\title{
Extensión cultural e ideología Las actividades de la Biblioteca de la Universidad de Zaragoza durante la Guerra Civil (1936-1939)
}

\section{(1) Luis Mariano Blanco Domingo \\ Universidad de Zaragoza, Departamento de Ciencias de la Documentación e Historia de la Ciencia, España Imblanco@unizar.es / https://orcid.org/oooo-0oo2-7492-4711}

\begin{abstract}
Resumen
El objetivo de este trabajo es analizar las tareas específicas en el ámbito de la extensión cultural que asumió la Universidad de Zaragoza (BUZ) durante la guerra civil española (1936-1939): la custodia y depósito de algunas colecciones particulares confiscadas; la participación en acciones de proselitismo ideológico y de afirmación patriótica; y por último, la tentativa frustrada de convertirse en una de las sedes de las muestras bibliográficas itinerantes de Italia y Alemania. Para ello, se han utilizado fuentes documentales procedentes de diversos archivos y consultado la bibliografía específica sobre el tema. Ello nos ha permitido concluir que las bibliotecas, y en especial la BUZ, se integraron plenamente en la maquinaria bélica de los bandos en conflicto, convirtiéndose en un arma propagandística eficaz.
\end{abstract}

\begin{abstract}
Ideological and cultural extension. The activities of the Universidad de Zaragoza Library during the Spanish Civil War (1936-1939). The aim of this article is to analyze the specific tasks in the field of cultural extension carried out by the Universidad de Zaragoza (BUZ) during the Spanish Civil War (1936-1939): The custody and deposit of some private collections confiscated; the participation in actions of ideological proselitism and patriotic affirmation; and finally, the frustrated attempt to become one of the headquarters of the itinerant bibliographical exhibitions in Italy and Germany. For this, documentary sources from various archives have been used and the specific bibliography on the subject was consulted. This has allowed us to conclude that the libraries, especially the BUZ, were fully integrated into the war machine of the sides in conflict, becoming an effective propaganda weapon.
\end{abstract}

Artículo recibido: 18-06-2018. Aceptado: 30-10-2018.
Palabras clave

Biblioteca de la Universidad de Zaragoza

Extensión cultural

Guerra Civil

Artigas, Miguel

Lasso de la Vega, Javier

\section{Keywords}

Universidad de Zaragoza Library Cultural Extension Civil Spanish War Artigas, Miguel Lasso de la Vega, Javier 


\section{Introducción}

El inusitado y celérico éxito del golpe de estado del 18 de julio de 1936 en Zaragoza, que supuso su rápida adhesión al bando rebelde, condicionó el ritmo institucional de todos los organismos públicos instalados en la ciudad. La Universidad, y por ende su biblioteca, no fueron una excepción. El centro, a través de su rector Gonzalo Calamita, cedió todas las instalaciones y medios disponibles para satisfacer las necesidades de los insurrectos, con la lógica parálisis de cualquier tipo de actividad académica.

Podemos establecer dos períodos claramente diferenciados en el devenir de las actuaciones de la Biblioteca durante la guerra civil. En el primero, que arranca con el inicio del conflicto y alcanza hasta marzo de 1938, observamos que las necesidades militares condicionan las decisiones y la adopción de cualquier medida. Una situación bélica confusa, con fronteras absolutamente permeables e indefinidas entre los dos bandos, confluye con la supremacía de lo militar y la escasa sensibilidad cultural de la oficialidad golpista, que consideraba el impulso que durante la República se dio a la difusión del libro una de las causas principales de la pérdida de los valores de la España imperial que perseguía y anhelaba. Las estructuras institucionales creadas por el Gobierno de Burgos, la Junta Técnica y las diferentes comisiones generadas, tenían en esta fase un carácter fundamentalmente instrumental y técnico, con el único objetivo de obtener el triunfo militar.

Durante esa etapa, la BUZ se convierte, de forma temporal, en el centro neurálgico de las decisiones relativas a la política bibliotecaria del bando nacional, y determinado por la lógica desconexión inicial provocada por un conflicto que se presumía rápido y exitoso para los instigadores del golpe de Estado, pero que sin embargo se dilató en el tiempo. Acoge además el control de la gestión bibliotecaria en la zona controlada por el bando sublevado gracias a la presencia accidental de las dos Inspecciones Generales de Bibliotecas y Archivos, encarnadas en las figuras de Miguel Artigas y Miguel Gómez del Campillo. Artigas, director de la Biblioteca Nacional y Presidente de la Junta Facultativa del Cuerpo de Archiveros, Bibliotecarios y Arqueólogos, asumirá la dirección sin que medie un nombramiento explícito.

El segundo período comienza en marzo de 1938, extendiéndose hasta la finalización de la contienda, determinado por la sustitución de la Junta Técnica como órgano de gobierno, por los ministerios, entre ellos el de Educación Nacional, la estabilidad del frente aragonés tras la derrota republicana, y la percepción de que la toma de Madrid era más inminente que nunca. En esta fase, en la que se intentan establecer los cimientos administrativos de un nuevo régimen ya inminente, se acelera la reorganización institucional del Cuerpo Facultativo de Archiveros, Bibliotecarios y Arqueólogos, confiada por Sáinz Rodríguez a Javier Lasso de la Vega. A partir de ese momento, y con Miguel Artigas desempeñando la dirección de los cursos para extranjeros de Santander y el cargo de bibliotecario del Instituto de España, la BUZ verá mermado su protagonismo, adaptándose a una actividad ordinaria siempre condicionada por unas circunstancias bélicas que la habían obligado a invertir parte de su escasa dotación económica en financiar los órganos creados ad hoc por las autoridades culturales golpistas.

Una de las facetas que más interés suscitó entre las autoridades del Nuevo Estado fue la utilización del patrimonio cultural como medio de propaganda y seducción, conscientes en parte de la necesidad de contrarrestar el enorme éxito que el gobierno republicano había obtenido en la defensa de su causa entre la intelectualidad europea (González Gallego, 1988; Saz Campos, 2013; Sevillano Calero, 2002). Para ello, desplegó una amplia batería de disposiciones que trataban de canalizar esa pretensión involucrando a las bibliotecas por un lado en la tarea de sometimiento y control ideológico de los fondos bibliográficos, y por otro en la 
búsqueda y/o reafirmación de apoyos externos. En las páginas que siguen pretendemos analizar su evolución y alcance en la sede zaragozana.

\section{Metodología}

Para la realización de este trabajo, junto con el aparato bibliográfico utilizado, hemos consultado numerosas fuentes primarias. Las relacionadas con los aspectos de la gestión de los fondos y la dinamización de las exposiciones proceden básicamente tanto del Archivo General de la Administración (AGA) como del Archivo de la BUZ, mientras que las informaciones relacionadas con las incautaciones de bibliotecas particulares y la represión del libro han sido extraídas de un fondo custodiado por el Departamento de Historia Contemporánea de la Universidad de Zaragoza. Todo ello sin desdeñar las interesantes aportaciones hemerográficas, fundamentalmente artículos publicados por el diario Heraldo de Aragón y la revista Universidad.

La historia social de la cultura, disciplina en la que se enmarca este trabajo, incorpora metodologías propias de la política, de la historia e incluso de la ciencia, para en este caso ofrecer un panorama de la instrumentalización de la cultura como factor de adoctrinamiento y vehículo de propaganda en el bando nacional. El concepto de "usos políticos" adquiere aquí una vertiente propia, que tiene como objetivos principales buscar la complicidad y los apoyos internacionales a la sublevación, y al mismo tiempo convencer (o a veces disuadir) a la población de la bondad de sus actuaciones, de la necesidad, en este caso, de resucitar la idea imperial de España como marco referencial.

\section{Estado de la cuestión}

El debate sobre la utilización de la cultura y las bibliotecas como elementos de propaganda y difusión ideológica en el bando nacional durante la guerra civil española ha gozado de cierto predicamento bibliográfico. La seminal obra de Alicia Alted (1984) es sin duda el primer referente fundamental, con su certero análisis sobre la necesidad de equilibrar, por parte de los sublevados, la enorme eficacia y alcance internacional de la estrategia desarrollada por el gobierno republicano. Pese a ello, las autoridades del bando nacional contemplarían con indisimulado recelo cualquier acción procedente de círculos "intelectuales".

Por otro lado, la relación entre cultura y política exterior ha sido objeto de estudio por parte de Lorenzo Delgado (1994) de una forma más global y no sólo con las potencias fascistas, Rubén Domínguez (2013) para el caso italiano, y sobre todo las investigaciones de Isabel Bernal Martínez (2007 y 2010) dirigidas a analizar la vinculación entre la Alemania nazi y la España nacional a través de las exposiciones bibliográficas.

\section{Las incautaciones y embargos de bibliotecas.}

Las circunstancias del conflicto motivaron la adopción de una serie de medidas destinadas a favorecer la consolidación de los principios ideológicos del bando nacional. Una de ellas está íntimamente relacionada con la visión que, en determinados círculos y dirigentes del mismo, se tenía del intelectual, a quien consideraban tanto el responsable directo de haber diseminado las ideas antipatrióticas que provocaron el movimiento militar que acabó con la República, como su vinculación con una política del libro abyecta y peligrosa, por cuanto se utilizó como vehículo de expansión de sus 
1. Como señala Alicia Alted. “El Nuevo Estado no ocultó su fuerte recelo hacia el mundo de los intelectuales y la cultura, pues a unos y otros atribuía las "odiosidades que han sido origen de esta guerra civil que padecemos". Alted, Alicia (1984: 253). En el mismo sentido, Jaume Claret manifiesta que "se acusaba a la intelectualidad española de haber introducido ideologías extranjeras desde cátedras y ateneos en detrimento de las esencias patrias, para acabar llevando al país a la decadencia a través de la instauración del régimen republi-

cano" Claret, Jaume (2006:21).

2. Orden de 10 de junio de 1938". Boletín Oficial del

Estado n 597 de 11 de junio

3. Gómez del Campillo había

asumido la doble condición de inspector de archivos y bibliotecas

merced al destino provisional de

Miguel Artigas a Santander para organizar el Curso para extranjeros desde la Sociedad Menéndez y Pelayo, que se iba a celebrar durante el verano de 1938 con el objetivo de buscar apoyos al bando franquista entre los intelectuales foráneos, y asumir su designación como bibliotecario del Instituto de España. 4. ABUZ. "Inspección realizada los días 4 y 6 de agosto de 1938 ". LibroRegistro de las Visitas de Inspección.

5. AHMC. Oficio del rector Gonzalo Calamita al General Jefe de la

Quinta División solicitando que los fondos de las bibliotecas particulares embargadas pasen a formar parte de las bibliotecas de las respectivas facultades. 17 de noviembre de 1936. Caja III. 6. AHMC. Carta del Rector al Decano de la Facultad de Medicina para que se persone en el domicilio de Jiménez Asúa para hacerse cargo de su biblioteca siguiendo órdenes del coronel jefe instructor Manuel Rodríguez Arnau. 13 de enero de 1937. Caja III

7. AHMC. Oficio del rector Gonzalo Calamita al General Jefe de la Quinta División reiterando la solicitud de que los fondos de las bibliotecas particulares embargadas pasen a formar parte de las bibliotecas de las respectivas facultades. 23 de febrero de 1937. Caja III.

8. AHMC. Relación de informes y acuerdos relativos a los interesados que se expresa en la comunicación adjunta relativos a las personas afectadas por resoluciones judiciales. 6 de noviembre de 1936. Caja III

9. Gumersindo Sánchez Guisande fue concejal del ayuntamiento de Zaragoza entre abril de 1931 y el mismo mes de 1933 elegido en las filas del Partido Republicano Radical Socialista. Para ampliar sus datos biográficos y su trayectoria intelectual puede verse López Casamayor (2007). ideas. La incautación y embargo de las bibliotecas de entidades y particulares se convierte por tanto en una necesidad quirúrgica y purificadora.

Cono salvaguarda y legitimación jurídica, la Orden de Vicepresidencia del Gobierno de 10 de junio de 1938 estipulaba la incautación de bibliotecas de organizaciones que formaron parte del Frente Popular o de personas a las que responsabilizaba de oponerse a la sublevación bien de forma directa o subsidiaria. ${ }^{2}$ El Inspector General Gómez del Campillo ${ }^{3}$ respondió que existían algunos procesos pendientes de resolución judicial, y que en cuanto se produjera el fallo de los mismos informaría debidamente al Ministerio. ${ }^{4}$ Se refiere a las bibliotecas particulares de Gumersindo Sánchez Guisande, Santiago Pi y Suñer y Felipe Jiménez Asúa, catedráticos de la Facultad de Medicina, requisadas por el Decano de la Facultad de Medicina Antonio Lorente Sanz siguiendo las instrucciones del Rector Calamita.

Sin embargo, la Universidad de Zaragoza ya había actuado previamente en ese sentido, impulsada por la implicación activa de su rector Gonzalo Calamita en apoyo de los sublevados. Un oficio del mismo, fechado el 17 de noviembre de 1936, y dirigido al General Jefe de la Quinta División, solicitaba que las bibliotecas de los encausados citados anteriormente pasaran a engrosar los fondos de las Facultades de la Universidad 5 . Meses más tarde ${ }^{6}$, ordenaba al Decano de la Facultad de Medicina, Antonio Lorente Sanz, que se personara en el domicilio de Jiménez Asúa con el objetivo de hacerse cargo de su biblioteca, siguiendo órdenes del coronel jefe instructor Manuel Rodríguez Arnau. El interés porque sus colecciones pasaran a formar parte de los fondos de la universidad era muy alto, a juzgar por una nueva carta enviada al General Jefe en la que se reiteraba la petición de que "sean puestos a disposición de la Universidad las existencias de libros que resulten en los procedimientos de embargo realizados por la autoridad judicial". ${ }^{7}$ En cualquier caso, los facultativos de la BUZ no intervinieron en ninguna de las fases del proceso, convirtiéndose en meros depositarios físicos de los volúmenes confiscados, y afirmando por tanto la exclusiva motivación política de las actuaciones.

El documento sobre las actividades de las personas afectadas por la resolución judicial, y que justificaba la confiscación de sus respectivas bibliotecas personales, se estructuraba en tres apartados: informe de la autoridad civil, informe judicial y por último acuerdo de la autoridad militar. ${ }^{8}$ En los tres casos este último concluye con un categórico: "Destitución", a pesar de los matices que presenta en el caso de algunos "encausados". Sánchez Guisande es definido como "radical-socialista", estrechamente relacionado con Pi y Suñer, y acusado de haber desempeñado el cargo de concejal republicano en el ayuntamiento de Zaragoza. ${ }^{9}$ Pese a su filiación ideológica "tiene sentimientos católicos, recta moral personal y profesional y sanas costumbres". La autoridad judicial, en la misma línea, considera que pese a dedicarse de forma activa a la propaganda y difusión de los programas electorales del Frente Popular, no puede demostrar ni afirmar sus concomitancias con "elementos revolucionarios", como parece deducirse de los rumores públicos.

En el inventario de su biblioteca ${ }^{10}$ encontramos 195 obras de medicina, y un bloque heterogéneo y multidisciplinar, que integra numerosas obras que muestran

10. AHMC. Relación de los libros de medicina y otras materias que procedentes de la biblioteca particular del catedrático D. Gumersindo Sánchez Guisande que se hace cargo con esta fecha y en representación del IImo. Sr. Rector de la Universidad de Zaragoza D. Antonio Lorenzo y Sanz, Decano de la Facultad de Medicina de la misma Universidad". Zaragoza 11 de enero de 1937. Caja III. 
su preocupación e interés por los temas políticos contemporáneos más candentes, y con una amplia representación de escritos pedagógicos de influencia institucionista. ${ }^{11}$

Pi y Suñer aparece definido igualmente que Sánchez Guisande como "radical-socialista" y ateo, pero también como hombre de sanas costumbres y recta moral. Procedente de la Institución Libre de Enseñanza, fue subsecretario de Instrucción Pública siendo ministro Barnés en el Gobierno de Azaña. La autoridad judicial proclama su decidida e inequívoca vinculación política con el Frente Popular, al que estaba afiliado siguiendo la estela del "funesto" D. Marcelino Domingo, quien fuera Ministro de Instrucción Pública durante el primer bienio republicano y en el primer gobierno surgido tras el triunfo del Frente Popular. Debido a su identificación ideológica, había desempeñado algunos cargos políticos. ${ }^{12}$

A Jiménez de Asúa por su parte se le identifica como "azañista después de ser radicalsocialista". Caracterizado como hombre de dudosa moral y ateo, también procede de la Institución Libre de Enseñanza. El juez estima que se ha demostrado su condición de socialista no sólo por su militancia sino por sus opiniones y apoyos directos a su hermano Luis, uno de los dirigentes más destacados de dicho partido. La mayor parte de su biblioteca contiene obras relacionadas con la medicina, destacando colecciones de revistas como Los progresos de la clínica, Spezielle Pathologie und Therapie innerer Krankheiten, o Paris Medicale. ${ }^{13}$

Sin embargo, el abandono y desinterés con que fueron tratadas las tres bibliotecas embargadas llamaron poderosamente la atención del inspector general Gómez del Campillo, quien demanda a Sánchez Viejo no sólo el inventario de las obras ingresadas en la BUZ a través de las medidas de embargo y enajenación dictadas según el
13. AHMC. Relación de los libros de medicina y otras materias que procedentes de la biblioteca particular del catedrático D. Felipe Jiménez de Asúa que se hace cargo con esta fecha y en representación del Ilmo. Sr. Rector de la Universidad de Zaragoza D. Antonio Lorenzo y Sanz, Decano de la Facultad de Medicina de la misma Universidad. Zaragoza 29 de enero de 1937. Caja III.

11. Entre ellas debemos destacar una buena colección de lecturas pedagógicas y sobre el desarrollo intelectual de la infancia (Pestalozzi, Johann Heinrich. Canto del Cisne, Leonardo y Gertrudis; Barnés, Domingo. El desenvolvimiento del niño, Preyer, W. El alma del niño, Menéndez Potenciano, Manuel. Fisiología de la educación, Decroly, Ovide. Educación infantil, Piaget, Jean El lenguaje y el pensamiento, Payot Educación de la voluntad, Montessori, María. Antropología pedagógica; Ferriani, Lino. Las mujeres y los niños en la vida social; Bunge, Carlos Octavio. Educación de los degenerados; Luzuriaga, Lorenzo. Programas escolares; Educación nueva; Kant, Pestalozzi y Goethe; Blanco, Rufino. Historia de la educación en España y América). Junto a ellas, convivían obras de institucionistas como Azcárate, Gumersindo de Estudios religiosos o Posada, Adolfo. Pedagogía con otras de autores conservadores como Aznar, Severino. Impresiones de un demócrata cristiano; o Maura, Gabriel. Bosquejo histórico de la dictadura; e incluso claramente fascistas como Vicente Gay o Giménez Caballero (Gay, Vicente. Las constituciones políticas: El verdadero gobierno de los pueblos. Giménez Caballero, Ernesto. Genio de España). Parecía además mostrar un gran interés por los aspectos políticos contemporáneos y el análisis del contexto histórico en el que se desenvolvía, a tenor de la presencia en su biblioteca de obras como Arenal, Concepción. Obras completas; Lamartine, Alphonse de. Historia de La Revolución francesa; Castelar, Emilio. Discursos parlamentarios; Darwin, Charles. Origen de las especies; González Blanco, Edmundo. Los sistemas sociales contemporáneos: colectivismo, anarquismo, sindicalismo, bolcheviquismo, exposición doctrinal comprendida; Gassote, Pierre. La revolución francesa; García Morales, Juan. 3 años de lucha a favor de los humildes; Pi y Margall, Francisco. Las nacionalidades; Garcitoral, Alicio. España en pie. La revolución de 1930 y otros ensayos; Madariaga, Salvador de. Ensayo de historia contemporánea; Roosevelt, Theodore. Mirando al porvenir; Lerroux, Alejandro. Al servicio de la República; Altamira, Rafael. Historia de España; Castro, Cristóbal de. Fariseos de la República; Ayensa, Emilio. Vista de la Causa seguida contra el señor Sánchez Guerra.

12. Santiago Pi Suñer fue Vicepresidente del Consejo de Instrucción Pública en 1931, y años más tarde Subsecretario del Ministerio de Instrucción Pública. Sin tener en cuenta las obras de Medicina, que alcanzan la cifra de 286 ejemplares incluyendo revistas y volúmenes facticios, en el inventario aparecen consignadas dentro del capítulo de "Obras varias" ejemplares de Descartes y el idealismo subjetivista moderno de Joaquín Xirau Palau; de Madariaga, Salvador de. Ensayo de historia contemporánea; Ingleses, franceses y españoles; La formación social de los trabajadores; Baudelaire, Charles. Les fleurs du mal; Ascarza, Victoriano F. Lecturas ciudadanas; Maeterlinck, Maurice. Le sablier; Ortega y Gasset, José. La España invertebrada; Andreiev, Leonid. El diario de Satanás; Azorín Lope en silueta; Maupassant, Guy de. Miss Harriet; Nitti, Francesco. La Europa senza paci; Bergamín, José. La más leve idea de Lope; Machado, Antonio. Poesías completas; Zweig, Stefan. Veinticuatro horas de la vida de una mujer; Amor; Ferry, Jules; René Descartes; La vie de Mahomet; Nietszche, Friedrich. Lo que los alemanes pueden perder; Azaña, Manuel. En el poder y en la oposición; Victor Hugo Les contemplations; López Rey, José. Los estudiantes frente a la dictadura; Kant, Immanuel La paz perpetua; Crítica de la razón práctica; Ciges Aparicio, Manuel. Del cautiverio; Wilde, Oscar Lord Arthur; The grieture of Dorian Gray; Leibniz El entendimiento humano; Remarque, Eva Marie Apres; Sombart, Werner. Lujo y capitalismo. También obras de Balmes, Donoso Cortés, Maurois o Romanones. AHMC. Relación de Las obras de medicina y otras materias que obran en la biblioteca particular de D. Santiago Pi y Suñer de que se hace cargo El Sr. Decano de la Facultad de Medicina D. Antonio Lorente Sanz, en representación del Exmo. Sr. Rector de la Universidad de Zaragoza. Zaragoza 28 de diciembre de 1936. Caja III. 
14. ABUZ. Oficio de Miguel Gómez del Campillo, Inspector General de Archivos e Interino de Bibliotecas y Museos a Pedro Sánchez Viejo, Jefe de la BUZ para que se encargue de los fondos bibliográficos incautados. Vitoria 5 de septiembre de 1938. Sin embargo, y pese a los esfuerzos de Gómez del Campillo, no parece que cuajara en exceso su preocupación por estimular el interés por la conservación de los ejemplares procedentes de las bibliotecas expurgadas Ello parece colegirse de un documento manuscrito de 1947, firmado por Ángel Canellas, relativo a los fondos que conserva

la Facultad de Filosofía y Letras, en el que señala que todavía en fecha tan tardía existen para papel viejo 30 vols., 87 folletos y 15 números de revista; para la Facultad de Medicina 13 vols. y 47 folletos, y quedan para la sección general 10 vols. y 24 folletos. ABUZ. Canellas, Ángel. Sobre fondos de las bibliotecas expurgadas.1947.

15. La revista Trébede publicó una serie de artículos en conmemoración de su centenario agrupados bajo el título Centenario de un exiliado. "Centenario de José Ignacio Mantecón” Trébede, 67, 2002, pp. 32-89. Torres H. Mantecón, Marco Aurelio (2004 y 2005). decreto 108 de 23 de septiembre de 1936, sino también una mayor preocupación por los fondos procedentes de las bibliotecas particulares de los catedráticos de medicina, "confuso montón de libros lastimosamente abandonados en un rincón del paraninfo ... obras que deberían ya estar en la biblioteca". Para evitar en lo sucesivo problemas de competencias jurisdiccionales con las autoridades militares o civiles, Gómez del Campillo le faculta, a través de un nombramiento oficial, para que reclame a quien corresponda la gestión de todos los fondos de libros existentes en otros locales, personas o entidades procedentes de incautaciones o embargos, sin que tal afán tuviese ninguna repercusión. ${ }^{14}$

También fue objeto de embargo la biblioteca particular del facultativo José Ignacio Mantecón Navasal, a quien el gobierno republicano nombró en 1937 Gobernador General de Aragón, hasta que en abril de 1938 se reincorporó al Ejército del Este como Comisario General. Se exilió primero a Londres y luego, tras una breve estancia en un campo de concentración en Francia, a México, donde orientó sus esfuerzos a la investigación y la docencia hasta convertirse en uno de los pilares de la biblioteconomía en México y Latinoamérica colaborando activamente con el bibliógrafo y archivero Agustín Millares Carlo. ${ }^{15}$

La colección quedó depositada en el Juzgado $n^{\circ} 3$ de Zaragoza hasta que la Comisión Central Administrativa de Bienes Incautados, tras haber ultimado el expediente de resolución, ordenara al Juez de la Comisión Provincial el 16 de diciembre de 1938 , previa comunicación a Miguel Gómez del Campillo como Inspector General de Archivos y Bibliotecas, que todos los libros incautados se trasladaran de forma definitiva a la BUZ sin ningún trámite ni documento de entrega, circunstancia que se produjo el 24 del mismo mes y año.

La biblioteca fue registrada después de la entrega en lista especial provisional para tener conocimiento del contenido. De la relación resultaron unos 1.085 asientos. Como las colecciones anteriores, en ningún momento se integraron con los fondos del establecimiento, permaneciendo depositadas y confinadas en un lugar aparte durante largos años. No será hasta agosto de 1959 cuando, gracias a la mediación de Mariano Baselga y de Marco Aurelio Torres, yerno de Mantecón, y después de interminables consultas y peticiones tanto ante el Gobierno Civil como ante el Rectorado, fueron entregados los volúmenes a Concepción de la Torre, esposa de Mantecón.

\section{Extensión cultural y propaganda política}

La conversión de la BUZ en un vehículo de transmisión ideológica condicionó el desarrollo de sus actividades. Ralentizadas o disminuidas las ordinarias, parte de sus esfuerzos se dirigieron a acatar y cumplir los distintos oficios y mandatos que perseguían el doble objetivo de fortalecer la cohesión en la retaguardia y difundir las bondades de las actuaciones sobre el patrimonio cultural realizadas por el bando nacional, buscando el contraste con la clara intencionalidad política y económica que, según ellos, tenían las autoridades republicanas.

\subsection{Las visitas a museos y monumentos.}

La Orden de 25 de abril de $1938^{16}$, inmersa dentro de la guerra de propaganda que sobre la responsabilidad en la destrucción del patrimonio monumental y cultural mantuvieron los dos bandos enfrentados, tras manifestar que una de las características más acusadas de la "barbarie roja" era la sistemática destrucción de monumentos y obras de arte, establecía que los funcionarios del Cuerpo debían organizar visitas semanales públicas y colectivas a sus museos y centros para explicar tanto la importancia y
16. “Orden de 25 de abril de 1938 organizando visitas colectivas a los Museos Arqueológicos y Monumentos Artísticos". Boletín Oficial del Estado $\mathrm{n}^{\circ} 555$ de 29 de abril. 
trascendencia histórica como el valor estético y artístico de las colecciones y piezas que en ellos se custodian. Justificaba tal decisión no sólo por la necesidad de estimular la atracción por las obras de arte y resaltar el mérito de sus creadores, sino también porque los Facultativos del Cuerpo debían intensificar su labor docente e incrementar su cercanía con la población para convertir su misión pedagógica en algo más vivo y real. Ello sin contar la pretensión didáctica y pedagógica que movía a los ideólogos del Nuevo Estado, uno de cuyos factores más sólidos consistía en divulgar y enfatizar el fuerte componente religioso que informa y preside las manifestaciones artísticas y la evolución histórica del pasado nacional.

La memoria de la BUZ de 1938 acredita la aplicación y cumplimiento de la orden, apoyando sin fisuras su firma y relacionándola con la creación de las Juntas de Cultura Histórica. Pese al notorio papel desempeñado por estas, justifica su aprobación no solo por la necesidad de difundir y divulgar las obras que constituyen el patrimonio documental de España, sino para crear la conciencia de que es preciso defenderlas para evitar su saqueo y destrucción. ${ }^{17}$

Pedro Sánchez Viejo, director de la Biblioteca, visitó a Mariano Pano y Ruata, Presidente de la Real Academia de Bellas Artes de San Luis, a fin de organizar conferencias y visitas a diversos monumentos de la ciudad para divulgar los tesoros artísticos que posee. El propio Presidente le comentó que el académico encargado del Museo tenía preparadas una serie de disertaciones sobre las colecciones existentes en el Centro. La organización de los actos y la estructura de los contenidos se postergaron hasta el curso siguiente, si bien la fecha de inauguración de las sesiones divulgativas se estableció para el 22 de diciembre de ese año.

En enero de 1939, el Cuerpo Facultativo de Archiveros, Bibliotecarios y Arqueólogos, siguiendo los preceptos que marcaba la disposición ministerial anteriormente mencionada, anuncia la celebración de un programa de conferencias sobre Historia del Arte español, acompañado de visitas a Museos y Monumentos de la ciudad. Seguía un criterio cronológico basado en los distintos períodos artísticos, y contenía 28 disertaciones que se iniciaban en la prehistoria y culminaban con un análisis de las manifestaciones coetáneas.

En algunas ocasiones, las conferencias iban acompañadas de visitas guiadas ilustrativas que pretendían divulgar la importancia y entidad de las obras analizadas. Así, tras su charla sobre el arte y la vida romana, Martínez Planells aprovechó las piezas del Museo Provincial para reforzar sus conclusiones, prestando especial atención al mosaico de Estada (Huesca). ${ }^{18}$ Velasco Taboada por su parte tuvo que exponer en dos sábados consecutivos, 18 y 25 de enero, debido a que la noche le sorprendió diseccionando las características esenciales del retablo mayor de la Seo. ${ }^{19}$

Sin embargo, este ambicioso repaso a la evolución del arte y su huella en Zaragoza experimentó algunos sobresaltos, inducidos generalmente por el exitoso curso de la guerra para el bando nacional. La conquista de Barcelona a finales de enero provoca el retraso de la conferencia de Albareda sobre el arte musulmán desde el 29 de enero hasta el 5 de febrero, ${ }^{20}$ y el definitivo triunfo militar el 1 de abril condiciona la celebración de las citas siguientes, hasta el punto que la última conferencia de la que tenemos noticia se celebró el 16 de abril, y tuvo como protagonista a Sancho Sala. ${ }^{21}$

\subsection{La Exposición del libro antiguo zaragozano (siglos XV al XVIII)}

Coincidiendo con las celebraciones de la Fiesta del Libro Español, el Patronato para el Fomento de Bibliotecas, Archivos y Museos Arqueológicos ${ }^{22}$ inauguró el 23 de abril de 1939, en el Palacio de la Diputación Provincial de Zaragoza, una exposición
17. "Ante los grandes infortunios de la patria desde el derrumbamiento de la Dictadura hasta después de las elecciones de Febrero de 1936, en que España se sintió conmovida por un huracán de odios que derivó en quema de templos, destrucción de monumentos y obras de arte, la Junta Técnica del Estado, por Orden de 23 de diciembre de 1936, creó en las provincias Juntas de Cultura Histórica y del Tesoro Artístico. Incalculable fue la labor cultural de estos organismos. Sin embargo, se notaba la falta de algo que contribuyera al conocimiento y conservación de nuestras obras de Arte, y el Excmo. Sr. Ministro de Educación Nacional dictó una orden, fecha 25 de abril de 1938 , disponiendo que se celebren con frecuencia visitas públicas a los Museos y Monumentos, partiendo de la base de que se trata de una labor metódica y objetiva de vulgarización de la Historia del Arte español para despertar en nuestro pueblo el amor a nuestros Monumentos, a estimarlos y defenderlos impidiendo su despojo y destrucción.” AGA. Educación. Memoria de la Biblioteca de la Universidad de Zaragoza 1938. Caja 31/5459.

18. "Conferencia del Sr. Martínez Planells sobre el arte y la vida romana". Heraldo de Aragón, 24 de enero de 1939.

19. "Conferencia del Sr. Velasco Taboada”, Heraldo de Aragón, 26 de enero de 1939.

20. "Conferencia del Sr. Albareda”, Heraldo de Aragón, 30 de enero de 1939.

21. "Conferencia del Sr. Sancho Sala", Heraldo de Aragón, 17 de abril de 1939.

22. Forman el Patronato entre otras personalidades de la vida cultural zaragozana el arquitecto Luis de la Figuera, Pedro Sánchez Viejo, Luis Boya, Bonet, Carlos Riba o Giménez Gran. Los cargos administrativos estaban ocupados por Mariano Burriel como secretario, el canónigo archivero Eduardo Estella como vocal, mientras que Miguel Allué ostenta el cargo de Vicepresidente. 
23. "La Fiesta Nacional del Libro en Zaragoza", Revista Universidad, 1939, $\mathrm{n} \div 2$.

24. Ibidem. No fue sencillo el acceso al puesto para Varela, puesto que el propio ayuntamiento manifestó ciertas reticencias aludiendo a que si un archivero que había ganado por oposición una plaza en el Ayuntamiento de Madrid tenía derecho a participar en el concurso, y si así lo fuera, si este título era suficiente aval para justificar su

presencia. Y ello pese a que alegó, entre otros méritos, la catalogación de todos los expediente de infanzonía (406) custodiados en el Archivo Histórico y de la Audiencia de Zaragoza. Para solventar el problema, Varela solicita la intercesión de Lasso de la Vega, no sin manifestarle que la verdadera causa del mismo era su condición de "extranjero" en la ciudad, lo que califica como "la tontería local". AHBMV. Fondo Lasso de la Vega. Carta de Eulogio Varela a Lasso de la Vega solicitando su intercesión para acceder al cargo de archivero municipal de Zaragoza. Zaragoza [s.f.]

Este resuelve de forma favorable a Varela, lo que permite su ingreso como archivero. AHBMV. Fondo Lasso de la Vega. Carta de Lasso de la Vega a Eulogio Varela resolviendo a su favor para el cargo de archivero municipal de Zaragoza. Vitoria, 3 de noviembre de 1938. AHBMV. Fondo

Lasso de la Vega. Tarjeta Postal de Eulogio Varela a Lasso de la Vega agradeciéndole su intercesión para que ocupara el cargo de archivero municipal de Zaragoza. Zaragoza, 8 de noviembre de 1938.

25. "Nuestro Movimiento exige la reivindicación de nuestra cultura nacional y nos encomienda la exensa tarea de formar los cerebros de nuestros hombres con sentido netamente español. El origen de esta cultura nacional es el libro. Nuestros mejores tiempos fueron aquellos en que conjuntamente peleábamos y escribíamos "La Fiesta Nacional del Libro en Zaragoza”, Revista Universidad, 1939, ํㅡ 2 .

26. Ibidem

27. "el gran libro de la sabiduría, el tesoro de todas las ciencias, el arca de todo consuelo, el centro de todo amor". Ibidem

28. "De los libros se han dicho muchas cosas: que son nuestros mejores amigos, que no nos engañan nunca, que son el alimento del alma ... pero estas fáciles sentencias no siempre son razonables y verdaderas, pues todas ellas parten de una base

falsa, a saber, que todos los libros son sanos y buenos". Ibidem monográfica sobre los incunables y libros raros producidos por los impresores aragoneses, que permaneció abierta al público hasta el 5 de mayo. ${ }^{23}$

Los contenidos procedían de los fondos de diversas instituciones: el Cabildo Metropolitano, la BUZ, el Real Seminario de San Carlos, el Seminario Metropolitano, el Ilustre Colegio de Abogados, la Diputación Provincial, el Real Seminario de San Carlos, el Archivo Histórico, la Real Maestranza de Caballería, y de las bibliotecas particulares de Miguel Allué Salvador, Mariano Baselga Jordán, María Jesús Corviño Arnau y César García Burriel.

La iniciativa y labores de coordinación se debieron a Eulogio Varela, archivero municipal de Zaragoza desde noviembre de $1938 .{ }^{24}$

En el acto de inauguración de la muestra pronunció el gobernador civil de la ciudad desde el mes de marzo, el carlista Antonio Iturmendi, un discurso sustentando en los parámetros ideológicos que marcaban el concepto que de la cultura y de la importancia del libro tenía el bando sublevado: la enfatización de su sentido nacional, su potencialidad como factor de adoctrinamiento y combate y la afirmación de la españolidad como necesaria y obligada norma de conducta. ${ }^{25}$

Simultáneamente a la muestra bibliográfica se celebró una sesión cultural en el salón de actos de la Facultad de Medicina, organizada por la Universidad de Zaragoza en colaboración con el Patronato. La presidencia fue ostentada por el general jefe de la Quinta Región Militar, Francisco Rañoy, quien compartió el estrado con el rector Calamita, el alcalde Juan José Rivas; el presidente de la Diputación Provincial y vicepresidente del Patronato de Archivos Miguel Allué Salvador; el coronel Benítez, jefe de Estado Mayor; el delegado de Hacienda Ramón Peñarredonda Fernández; el presidente de la Audiencia Gerardo Álvarez de Miranda; por la Jefatura Provincial de Falange Joaquín de Pitarque y Elío; y en representación del arzobispo Juan Garriga.

La apertura del acto fue responsabilidad del Rector Calamita. A continuación el secretario del Patronato Provincial de Bibliotecas, Mariano Burriel, dio lectura de la Memoria anual de la institución, en el que resumió la labor desarrollada durante ese año, acompañada de una estadística de los libros donados por los zaragozanos con destino a la Exposición inaugurada". ${ }^{26}$ Tras ello, realizó un esbozo de los proyectos aprobados en el marco del objetivo de procurar todos los medios para popularizar la cultura.

Siguió en el orden de intervención la lectura de un trabajo sobre el Quijote y las novelas ejemplares cervantinas por parte del bibliotecario del Sindicato de Estudiantes Universitarios (SEU), José Serichol, quien aprovechó su presencia en la tribuna para ensalzar la trayectoria de su institución y defender la necesidad de crear bibliotecas populares.

Miguel Allué por su parte comienza su disertación proclamando la centralidad de la religión en todo tipo de actividad científica y literaria, simbolizada en el crucifijo presidencial. ${ }^{27}$ Pero el mensaje principal que transmite tiene como objetivo reforzar la tesis de que es fundamental la apuesta por la lectura dirigida y selectiva, puesto que no todos los libros resultan edificantes. ${ }^{28}$ Con una pretensión claramente divulgadora y fuertemente paternalista, enumera algunos ejemplos que le sirven para concluir que los libros de la nueva España no sólo han de llegar al corazón de los españoles, sino que deben "hacernos soñar dulcemente en la grandeza de nuestra Patria". ${ }^{29}$

En representación de la Universidad, Álvaro San Pío diserta sobre Sugestiones curiosas sobre libros y lectores, una propuesta levemente alejada de la retórica triunfalista 
imperante y más cercana a elementos puramente científicos que recoge las características esenciales de conceptos como bibliofilia, biblioteconomía, bibliografía, bibliomanía y bibliopiratería. No obstante, tampoco escapa al maniqueísmo interesado que considera la existencia y rivalidad constante entre los libros perniciosos y los "buenos".

La BUZ elaboró un primer listado de obras a exponer que los organizadores no contemplan en su totalidad, bien porque algunas coinciden con las aportadas por otras instituciones o particulares, o porque el comité decide, en última instancia, realizar un filtrado más amplio. El catálogo final está formado por 68 títulos, de los cuales 17 son los que corresponden a la BUZ.

Las obras elegidas constituyen una selección muy representativa de la riqueza bibliográfica que atesoraba la biblioteca, y que guarda estrecha relación con la enorme importancia que la ciudad de Zaragoza tuvo para la difusión de la imprenta y el desarrollo industrial del libro en España. Destacan el Manipulus curatorum (1475), debida al taller de Mateo Flandro y primera obra impresa en Aragón, y otros dos incunables, Fori aragonum (1496) y la Crónica de Aragón (1499), obra de Gauberto Fabricio de Vagad, ambos impresos por Pablo Hurus. Se incluye además en el mencionado anexo una relación de 12 obras que no se incorporaron a la muestra por diversos motivos que se señalan.

\subsection{Las nonnatas muestras bibliográficas de Italia y Alemania}

La debilidad y escaso éxito de la labor propagandística del bando nacional entre los intelectuales foráneos y, como contrapunto, la mayor fortuna y capacidad de penetración de la política cultural exterior por parte de las autoridades republicanas, con la mayoría de los países reticentes a reconocer diplomáticamente al incipiente Estado de Burgos hasta casi finalizado el conflicto bélico, propiciaron el impulso entre los dirigentes del bando sublevado de una serie de iniciativas que mitigaran o redujeran el alcance y difusión de ambas situaciones..$^{30}$ Así, fueron constantes las visitas al extranjero con el objetivo de divulgar las razones que habían provocado la insurrección armada, la organización de viajes para periodistas e intelectuales foráneos a la España nacional para que mostrasen una imagen positiva de la zona bajo su control, o la celebración de congresos y cursos para extranjeros que sirvieran de testimonio de las bondades del Nuevo Estado y legitimaran el recurso a la fuerza para desarbolar a la República.

Para facilitar tales actuaciones, surgieron mecanismos institucionales que intentaran canalizar y organizar los eventos destinados a tal efecto. La creación del Instituto de España pretendía servir de centro difusor de ideas reuniendo a todos los intelectuales españoles afectos al nuevo régimen.$^{31}$ Pero es sin duda el restablecimiento de la Junta de Relaciones Culturales con el primer gobierno de Franco en 1938 el mejor exponente de esta perentoria necesidad de recabar apoyos culturales más allá de los aportados por sus aliados naturales, Portugal,y sobre todo Italia y Alemania. ${ }^{32}$

Uno de los elementos característicos de la política cultural del bando nacional, junto a la simbiosis entre cultura y propaganda, es su absoluta supeditación a la política exterior, que en el caso de Italia y Alemania adquiere tintes extremos. La propia dinámica del conflicto y la situación de orfandad internacional en la que se encontraba el todavía embrionario estado franquista, motivaron que el único recurso y apoyo foráneo procediera de las potencias europeas fascistas, más afines ideológicamente, y que prestaron apoyo material y militar en pro de la causa nacional. Tanto Hitler como Mussolini aprovecharon esta coyuntura para influir en la formación de los
30. Alicia Alted llega a hablar de “obsesión por contrarrestar de manera positiva los argumentos propagandísticos republicanos" cuando se refiere a la labor de Pedro Muguruza al frente de la Comisaría General del Servicio de Defensa del Patrimonio Artístico Nacional desde 1938, y basa su argumentación en el contundente y crítico informe que envía al ministro Pedro Sáinz Rodríguez, en el que expone:"...pude ver como en el extranjero, en los centros culturales de arte y de historia, estamos conceptuados a un nivel muy inferior del otorgado a los rojos ... pude también comprobar que en el orden artístico, nuestras propagandas, sólo apoyadas en la labor negativa de los rojos, son contraproducentes" Cartainforme a Pedro Sáinz Rodríguez en Alted, Alicia (1984: 92).

31. La creación del Instituto, idea original de Eugenio d'Ors para agrupar a los miembros de las Reales Academias dispersos en la zona nacional, pretendía otorgar, en palabras de Sáinz Rodríguez, “el matiz cultural al Alzamiento ante los ojos de Europa" Alted, Alicia (1984: 239). Sin embargo, el proyecto carecía de contenido y competencias, por lo que sus realizaciones fueron prácticamente nulas, máxime teniendo en cuenta que pocos meses después de finalizada la guerra, el nuevo ministro de Educación Nacional Ibáñez Martín crearía el exitoso Consejo Superior de Investigaciones Científicas (CSIC).

32. Para profundizar en la relación entre política cultural del bando nacional y su política exterior, véase Delgado GómezEscalonilla, Lorenzo (1994). 
33. Ibidem

34. AHBMV. Fondo Lasso de la Vega. Carta de Javier Lasso de la Vega al Rector de la Universidad de Zaragoza sobre la exposición del libro italiano. Vitoria, 18 de noviembre de 1938.

35. AHBMV. Fondo Lasso de la Vega. Carta de Gonzalo Calamita a Javier Lasso de la Vega sobre la exposición del libro italiano. Zaragoza, 23 de noviembre de 1938.

36. Bernal Martínez se hace eco de las fricciones provocadas por conflictos de competencias entre una Falange que dirigía la censura y el sector católico conservador que controlaba la gestión de las bibliotecas a través del ministro Sáinz Rodríguez. (Bernal Martínez, Isabel, 2010)

37. ABHMV. Fondo Lasso de la Vega. Carta de Lasso de la Vega a Gonzalo Calamita sobre la exposición del libro alemán. Vitoria 9 de enero de 1939.

38. AHBMV. Fondo Lasso de la Vega. Carta de Gonzalo Calamita a Javier Lasso de la Vega sobre la exposición del libro alemán. Zaragoza, 16 de enero de 1939. cuadros de mando del futuro Estado merced a los cada vez más constantes proyectos conjuntos establecidos con la Junta. ${ }^{33}$

El interés por celebrar exposiciones bibliográficas que ofrecieran una visión panorámica de los logros obtenidos por Italia y Alemania dio lugar a una serie de múltiples reuniones y contactos que, por diversas causas, no fructificaron. Pese a la obstinación y el trabajo de Miguel Artigas y Javier Lasso de la Vega, ni la apuesta por una muestra circulante y móvil acompañada de disertaciones multidisciplinares apologéticas de las bondades del fascismo, ni una exposición proyectada en Santander pudieron llevarse a cabo.

La Universidad de Zaragoza también recibió una comunicación de Lasso de la Vega el 18 de noviembre de 1938 , en la que informa del encargo realizado desde el Ministerio de Educación Nacional para organizar una exposición del libro italiano conjuntamente con la embajada del país transalpino. ${ }^{34}$ El rector Calamita designa como Presidente a Pascual Galindo, y como lugar de celebración la Casa del Fascio. ${ }^{35}$ Sin embargo, como en los casos anteriores, no pudo desarrollarse por la escasa implicación y desinterés de las autoridades franquistas.

La realidad fue que, a pesar de estos conatos, la principal exposición itinerante del libro italiano en España tuvo lugar tras finalizar la guerra civil, destacando especialmente la celebrada en Santander en agosto de 1942, aprovechando la celebración de los cursos universitarios de verano (Domínguez Méndez, 2013).

Lasso fue el encargado de firmar el acuerdo que el 14 de octubre de 1938 se estableció entre el Deutsch-Ausländischer Buchtausch y el Servicio Nacional de Archivos y Bibliotecas del gobierno de Burgos, con el objetivo de incentivar la relación cultural con la Alemania nazi a través de iniciativas como las donaciones de libros alemanes, la participación de intelectuales españoles en congresos, o la organización de exposiciones de libros (Bernal Martínez, Isabel, 2007).

La proyectada muestra itinerante de libros alemanes sufrió diversos avatares, aunque contribuyó a la propaganda política del nacionalsocialismo y a la sustitución de los fondos bibliográficos de los centros superiores de educación e investigación españoles con un doble objetivo: neutralizar en la medida de lo posible la influencia francesa y difundir un bloque de doctrina dirigida, de raíz filogermánica, que coadyuvará a asentar las bases teóricas del Nuevo Estado desde una perspectiva nacionalsocialis$t^{36}$. Oficialmente, en su periplo por territorio español, no se contemplaba Zaragoza como sede. Sin embargo, Lasso recaba la opinión de Calamita sobre la posibilidad de celebrarse en la capital del Ebro ${ }^{37}$, obteniendo una entusiasmada respuesta del Rector quien, siguiendo los deseos del agregado cultural de la embajada alemana, Wilhelm Petersen, habilitaría el Paraninfo de las Facultades de Medicina y Ciencias como marco adecuado. Y no conforme con la mera aceptación, le envía la relación de los catedráticos que integrarían el hipotético comité organizador: Domingo Miral, Pascual Galindo, Leonardo Prieto y Sancho Seral, a reserva de otros profesores que quisieran sumarse. ${ }^{38}$ Sin embargo, el intento fracasaría.

\section{A modo de conclusión. La biblioteca como arma de combate}

La BUZ asumió como una de sus funciones más destacadas durante el conflicto bélico erigirse en vehículo de transmisión ideológica del bando sublevado. Para ello no sólo adaptó y modificó los servicios ordinarios, sino que además transformó las actividades de extensión cultural siguiendo las directrices emanadas de las disposiciones legales dictadas por el mismo. 
En primer lugar, fue el destino de las confiscaciones de bibliotecas particulares, si bien la incorporación de estos fondos fue más nominal que efectiva. La mayor parte de los mismos permanecieron en custodia y ajenos a la actividad bibliotecaria, sin integrarse ni relacionarse con la colección ni siquiera desde el punto de vista del proceso técnico, ya que los facultativos apenas intervinieron en ninguna de las fases.

En segundo lugar, al mismo tiempo que se establecieron las bases teóricas y legales para la depuración de las obras consideradas nocivas y peligrosas, el gobierno de Burgos articuló una serie de medidas que buscaban difundir una imagen positiva de los logros y avances alcanzados en la defensa del patrimonio cultural en el territorio que controlaba jurisdiccionalmente. Y lo hizo prestando atención a dos aspectos fundamentales: las visitas a los museos y monumentos de las ciudades y la organización de exposiciones bibliográficas. En el distrito zaragozano se desarrolló una importante labor de divulgación en ambos sentidos, creando un completo ciclo de conferencias y visitas a los monumentos más representativos, y organizando una exposición sobre el libro antiguo impreso en Zaragoza. Las iniciativas no sólo se transformaban en instrumentos que aunaban ocio y propaganda, sino también en un hábil intento de revitalizar el protagonismo de los funcionarios del Cuerpo Facultativo y reivindicar la necesidad de su presencia.

La más importante de las muestras bibliográficas en las que participó la BUZ fue la dedicada al libro antiguo impreso en Zaragoza. Este intento de recuperar el vigor e importancia del fondo antiguo de las bibliotecas universitarias ubicadas en el territorio controlado jurisdiccionalmente por los rebeldes obedece en parte a la reivindicación de un pasado glorioso utilizado para justificar y legitimar el levantamiento militar, y a su catalogación y accesibilidad dedicaron gran parte de sus desvelos los facultativos adscritos temporalmente a la biblioteca.

El mismo efecto pretendían alcanzar las exposiciones bibliográficas selectivas y monográficas sobre las potencias del eje que apoyaron el golpe de estado, Italia y Alemania. Sin embargo no pasaron de la planificación teórica por diversas circunstancias pese a los esfuerzos de Lasso de la Vega o Miguel Artigas.

La actividad bibliotecaria de la BUZ quedó por tanto supeditada al devenir de la guerra, teñida de propaganda política, y plegada a los nuevos parámetros ideológicos del Nuevo Estado, que conferían al libro una labor secundaria, cuando no perniciosa, siempre que no estuviera dirigido y controlado. La apelación al proselitismo ideológico como método para asegurar y/o mantener la cohesión social de una ciudad de retaguardia como Zaragoza, la búsqueda de una mayor resonancia y apoyo al bando franquista en los medios públicos internacionales, y un intento de reivindicar la importancia del Cuerpo Facultativo de Archivos, Bibliotecas y Museos son los rasgos más representativos de la extensión cultural bibliotecaria.

Con este trabajo hemos analizado un modelo de extensión cultural específico, el de la BUZ, perfectamente integrado en la planificación de la propaganda del bando nacional, que respondía a las especiales circunstancias que concurrían en una ciudad de retaguardia, y durante algún tiempo convertida en "capital" bibliotecaria de los sublevados gracias a la presencia y actividad de Miguel Artigas. 


\section{Q Referencias bibliográficas}

》Alted, Alicia. 1984. Política del Nuevo Estado sobre el patrimonio cultural y la educación durante la Guerra Civil Española. Madrid: Dirección General de Bellas Artes y Archivos, Centro Nacional de Información Artística, Arqueológica y Etnológica.

» Bernal Martínez, Isabel. 2007. Libros, bibliotecas y propaganda nazi en el primer franquismo: las exposiciones del libro alemán. En Hispania nova. Revista de Historia Contemporánea. No. 7. <http://hispanianova.rediris.es/7/articulos/7ao1o. pdf> [Consulta: 4 agosto 2015].

» Bernal Martínez, Isabel. 2010. La Buchpropaganda nazi en el primer Franquismo a través de la política de donaciones bibliográficas (1938-1939). En Ayer. No. 78, $195-232$.

»Claret Miranda, Jaume. 2006. El atroz desmoche: la destrucción de la universidad española por el Franquismo, 1936-1945. Barcelona: Crítica.

"Delgado Gómez-Escalonilla, Lorenzo. 1994. Las relaciones culturales de España en tiempo de crisis: de la II República a la Guerra Mundial. En Espacio, Tiempo y Forma. Serie V, Historia Contemporánea. No. 7, 259-294.

»Domínguez Méndez, Rubén. 2013. Apuntes sobre la exportación del libro italiano en España durante el ventennio fascista. En Ogigia. No. 14, 37-49.

»González Gallego, Isidoro. 1988. Falange y educación: Zaragoza, 1936-1940. En Historia de la Educación: Revista Interuniversitaria. No. 7, 203-230. <http:// dialnet.unirioja.es/servlet/articulo?codigo $=87406$ \&orden $=279499$ \&info=link $>$ [Consulta: 12 diciembre 2014].

"López Casamayor, José Luis. 2007. Gumersindo Sánchez Guisande: maestro de enseñanza y de vida. Almonacid de la Sierra: Ayuntamiento de Almonacid de la Sierra.

» Saz Campos, Ismael. 2013. Falangistas y católicos reaccionarios: una batalla político-cultural decisiva. En Mateos, Abdón, ed. Las dos caras del fascismo. Madrid: Comares. p. 135-146.

"Sevillano Calero, Francisco. 2002. Propaganda y dirigismo cultural en los inicios del nuevo Estado. En Pasado y memoria, revista de historia contemporánea. No. 1, 81-110.

» Torres H. Mantecón, Marco Aurelio. 2004. Semblanza de José Ignacio Mantecón Navasal. En Educación y biblioteca. Vol. 16, no. 139, 74-81. <http://gredos. usal.es/jspui/bitstream/10366/119042/1/EB16_N139_P74-81.pdf>. [Consulta: 12 abril 2015].

»Torres H. Mantecón, Marco Aurelio. 2005. José Ignacio Mantecón. Vida y obra de un aragonés del destierro. Zaragoza: Institución Fernando el Católico. 